\title{
Theoretical foundations of the diagram method for calculating rod elements made of reinforced concrete
}

\author{
Oleg Radaykin* \\ Kazan State Power Engineering University, 420066, Kazan, Russia
}

\begin{abstract}
At the present stage of development, the diagram method for calculating rod elements made of reinforced concrete has a number of disadvantages, which is due to the lack of a single holistic theory. The paper attempts to propose its foundations. For this purpose, the most General structure of the theory has been developed, and a three-level computational model has been proposed, hierarchically built on the principle of the computational scheme discretization: level 1 - "element" the rod design model, built according to the well-known rules of construction mechanics; level 2 - "cross" - enhanced non-linear (discrete) deformation model, adopted by $\mathrm{BC} 63.13330$ with some significant author's clarification; level 3 - material - the standard samples' analysis model of concrete and rerod subject to the axial compressive action or tensile static short-term load or under static short-time load, pure shear (to describe proposed Jenny more deformation curve of concrete). A detailed algorithm for the relationship of models at all three levels is described.
\end{abstract}

\section{Introduction}

The main result of the calculation that ensures the structural safety and operational suitability requirements for any structure or element is to obtain their stress-strain state (SSS). This requires a reliable determination of forces and displacements (stresses and strains) at all points of the element, depending on the load. In this case, reinforced concrete from a certain level of loading begins to show physical nonlinearity, both due to concrete and reinforcement plasticity, and due to cracking, as well as other factors (geometric and structural nonlinearity, creep and shrinkage are not considered in this work). In this regard, for the SSS description at any point or section, the corresponding de-formation diagrams of materials (concrete and steel) are usually used. These diagrams as part of a nonlinear deformation model, which also includes the equilibrium equations, deformation compatibility and boundary conditions, constitute the essence of one or another diagrammatic method for calculating reinforced concrete. Each of them has its own characteristics depending on the type of diagram used and the solution algorithm, but they all have the fact that each of them is the same for calculating the structure both for the first and second groups of limit states, and, in addition, gives a possibility to estimate SSS at all

\footnotetext{
*Corresponding author: olegxxii@mail.ru
} 
stages of its loading - from zero up to destruction. This all determines the advantage of diagrammatic methods over traditional calculations for ultimate forces and permissible stresses, since the latter include semi-empirical simplifications and give an unacceptable error (usually in reserve), have a limited scope, and contain a lot of experimental coefficients with an incomprehensible physical meaning.

In its most general form, however, the use of diagrammatic methods is analytically difficult to implement. In this regard, in practice, they resort to solving problems in numerical form, which can be automated at electronic machines. The appropriate discrete deformation models are used for this.

At the same time, we note that the most common means of automating calculations today is the finite element method (FEM). However, in a number of cases, when solving nonlinear problems of reinforced concrete mechanics, its use is not always convenient for the following reasons:

- it is necessary to master a specialized software complex and, in some cases, not one, but several - depending on the range of the tasks to be solved;

- often the user does not have the opportunity to make his own changes to the calculation apparatus of the software complex, both due to the closed nature of the program code, and due to lack of knowledge of special programming languages (for example, APDL the language in Ansys);

- in most cases, the designer in practice deals with the structures, which work under load is determined by one (maximum two) dangerous sections (for example, for a hinged beam - one normal section in the middle of the span and one inclined in the support zone), therefore, in more detailed assessment SSS the rest of the sections are not necessary (and this would reduce the time and labor costs for modeling and analyzing the results).

Therefore, the best option would be to use FEM as a basis for obtaining efforts in the section under consideration, while remaining within the linear theory boundaries. For this, any software package that implements FEM and designed for the calculation of building structures may be useful.

And directly for the nonlinear calculation of a reinforced concrete section, the most suitable, in the author's opinion, is the numerical diagrammatic method based on the socalled nonlinear deformation model, adopted in BC 63.13330 (and earlier in SNiP 52-012003). Nevertheless, the field of this method application is very limited today, attempts to extend it to a wider class of rod structures and elements under different loading conditions are only advisory and very conditional. The regulatory documents, which include this method as an alternative to the method of limiting efforts, do not take into account many significant factors affecting the reinforced concrete rods' operation, and, moreover, do not contain specific numerical examples that clearly demonstrate the algorithm of methods for its basis.

The listed disadvantages and imperfections of the numerical diagrammatic method are primarily due to the lack of a unified integral theory. In this work, an attempt to determine its foundations is made.

\section{Models and Methods}

We will introduce a number of definitions for a deeper understanding of the material presented.

Theory - it is a set of interrelated concepts (both abstract and specific) that are specific for a given specific field of research and a specific scientific discipline, as well as algorithms, which allows: 
a) to predict (for example, to solve the problem of the most reliable assessment of structural safety and operational -the suitability of a building, structure, element or unit),

b) explain,

c) describe a phenomenon (process).

The structure of any theory, according to the author, should include the following:

1. A set of specific concepts in their original form:

a) concept - the essence, the main idea of the theory;

b) research object and theory scope (definition of general and particular cases of application);

c) primary empirical basis, obtained on the basis of measurements using an instrumental base or speculatively;

d) initial premises, assumptions, hypotheses, axioms, postulates, etc.

e) terminology.

2. A set of specific algorithms in their original form: methods, techniques, principles, rules, canons.

3. Intersection of specific concepts and algorithms - the models.

Model - it is a set of the modeled object image (information) and the algorithms of its behavior and transformation when interacting with other objects, captured on a material medium different from the original object (the definition was introduced by the author for the first time).

Where in:

1 - the model provides a more convenient and accessible study of the original object than its direct consideration (idealization);

2 - the original object image and algorithms' detail degree depends on the problem conditions (abstraction);

3 - the transition from the original object to the model is carried out according to the rules of the similarity theory (the manifestation of large-scale effects);

4 - the image and algorithms of the original object and the model have a one-to-one correspondence and are described in an easy-to-understand language (in a coding system), for example, the language of mathematics, logic, structural mechanics, etc.;

5 - a piece of paper (drawings, diagrams), neurons of the human brain (psyche), crystals of microcircuits and a computer display, etc., can act as a material carrier.

In the mechanics of a solid deformed body and in the theory of reinforced concrete in particular, the model most often uses the so-called independent (otherwise "ideal" independent of the carrier material) models, which are usually presented in the form of a modeled object calculation scheme (i.e., its image) and the mathematical apparatus describing the algorithms for changing and transforming this calculation scheme in interaction with the external environment. In this case, the design scheme includes a geometric diagram in the form of a drawing idealizing an object, a diagram of loads, internal forces, stresses and deformations. The mathematical apparatus traditionally consists of equilibrium equations, deformation compatibility conditions, physical relations, as well as boundary conditions.

Modeling - this is a study of the modeled original object by:

1) building its model,

2) studying its properties when interacting with other objects,

3) transferring the received information (information and algorithms) to the modeled object.

As already mentioned above, the developed theory basis, as, indeed, any other, should be a model, within the framework of the terminology adopted in [1], in the context of the issue under consideration - this will be a computational model. 
It is accepted, depending on the initial prerequisites, to divide all design models of the reinforced concrete strength resistance into integral and discrete ones, which are the basis, respectively, of the analytical and numerical varieties of a particular theory (method). As noted by V.M. Bondarenko [2], "objectively in content and chronologically in time, discrete models are hereditary in relation to integral ones".

An analysis of the models features of both types can be extracted from the work of V.S. Fedorov, V.E. Levitsky [3], this analysis is partially given below with the additions and clarifications of the author of this work.

So, in integral models, the sections of a reinforced concrete element are represented as a set of a relatively small number of components, within which a certain function of changing stresses or deformations is set. These models include the model of integral estimates by V.M. Bondarenko [2], in which the integral modulus of concrete deformation is introduced. It is effective only for the relatively simple sections' calculation, while the introduction of additional components, for example, a steel shell, or SSS complication, for example, up to oblique bending, significantly "makes the model heavier" to its analytical use impossibility. Integral models of A.B. Golyshev and V. Ya. Bachinsky [4], B.S. Rastorguev [5], A.V. Borovskikh and V.G. Nazarenko [6], V.I. Kolchunov and A.I. Nikulin [7] and others are also known. They are based on specifying the stress state of concrete by substituting the law of deformation change along the section height into the physical ratio. As a result of the stress diagrams integration, the resultant forces in the compressed and stretched zones are determined, which then participate in the equilibrium equations. This approach does not allow taking into account the gradient effects of the concrete force resistance in an inhomogeneous stress state.

The integral approach has been significantly developed in the so-called block models, in which reinforced concrete elements with cracks are represented as a system of elastic blocks interacting with each other along the compressed zone and tensile reinforcement. Such models are considered in the works of H.M. Westergaard, A.A. Gvozdev, Yu.V. Zaitsev, E.N. Peresypkin, V.I. Kolchunov and others. Block models can significantly expand the range of tasks to be solved, however, their numerical implementation is difficult due to insufficient knowledge of the concrete cracking process. The model described in [8] deserves special attention.

In discrete models, a section is presented as a set of a fairly large number of components, within each of which stresses, deformations, and material properties are assumed constant. Each component is considered to operate under axial compression or tension, and its behavior is characterized by a corresponding deformation diagram. This approach makes it possible in a fairly simple way to take into account the structural heterogeneity, physical nonlinearity of materials, the formation and development of cracks, and thus implement the general case of calculating various types of reinforced concrete rod elements with an arbitrary cross-sectional configuration, with different conditions of eccentric load application, etc.

At the same time, we note that integral models assume only analytical calculation of structures and discrete ones only numerical, which, of course, is more convenient from the point of view of automation and programming.

Efforts, stresses, deformations, displacements can act as control and managing parameters in models of both types. So, for example, the normative method of limiting forces uses, the previously used method for permissible stresses can be presented as control and managing parameters. The models where deformations are used as such parameters are called deformation models. Thus, the modern diagrammatic method in BC 63.13330 is based on a discrete deformation model. Its historical name is nonlinear deformation model. Integral deformation models are used, for example, in the deformation theory of plasticity (the theory of small elastoplastic deformations according to A.A. Ilyushin) and flow theory. 
The development of discrete deformation models of reinforced concrete resistance in our country is devoted to the research of V.N. Baikov, M.I. Dodonov, B.S. Rastorguev [9]; N.I. Karpenko [10], [11]; A.S. Zalesov, T.A. Mukhamediev, E.A. Chistyakov [12] and others. Models of this type are included in the design standards for reinforced concrete structures in the EU, USA, Japan, Russia, Ukraine and Belarus.

As already mentioned, in order to develop and improve the normative discrete deformation model, it is necessary to create a certain theory based, one way or another, on the postulates, principles and hypotheses of the rigid deformable body mechanics. At the same time, of course, in some cases, the researcher can use another theoretical basis - from any adjacent direction of physics - electrodynamics, hydraulics, etc. This is possible due to the similarity of the considered solids deformation processes and, for example, the interaction processes of electric current stress fields. This case is not considered in this study.

The mechanics of a solid deformable body, taking into account the specifics of reinforced concrete, uses the following basic hypotheses and principles, which, among other things, are applied to develop the proposed theory:

1 - the hypothesis about the smallness of the "elementary" volume of the body in comparison with its general dimensions; within the boundaries of this volume, the material properties, as well as the parameters of its force interaction with other volumes of the body and the external environment, can be considered homogeneous (invariable in volume); the hypothesis makes it possible to apply both analytical and numerical apparatus of differential and integral calculus to solve the problems of mechanics;

2 - the hypothesis of the material continuity, which is often used in the form of a condition on the reinforced concrete components' deformations compatibility; according to it, the material occupies the volume of the body completely - without breaks and voids, which allows using the theory of continuous functions;

3 - the hypothesis of plane sections (J. Bernoulli): the sections that are plane and normal to the rod axis before deformation remain plane and normal to its axis and after deformation - simplifies the relationship between the deformation field components in the cross section of the rod to a linear law;

4 - the assumption that the rod cross-section dimensions are small in comparison with its length; allows you to use the mechanics of rod systems to find internal forces in the rod cross-section;

5 - the centerline of the rod is considered inextensible;

6 - the principle of Saint-Venant is valid, which asserts that different, but statically equivalent, local loads cause the same stress state in the rod (if local stresses near the point of load application are not taken into account);

7 - hypothesis about the unstressed initial state; in accordance with this hypothesis, there are no internal forces in an unloaded body;

8 - laws of energy and mass conservation for the closed systems (isolated bodies);

9 - fundamental variational principles of a solid deformed body mechanics, which determine the true stress-strain state of a loaded body among all its possible states according to the criterion of minimum or maximum of a certain physical quantity (for example, strain energy);

10 - general principles of calculation and design of structural elements made of reinforced concrete:

10.1) compliance of the design with operating conditions;

10.2) the least laboriousness of manufacturing;

10.3) lowest installation speed;

10.4) saving materials (concrete and steel); 
10.5) combination of functions: savings are achieved if one element performs several functions;

10.6) the material concentration (for example, this is the use of a curved rod shape in accordance with the distribution of forces and stresses in it);

10.7) the principle of the design scheme discretization and its presentation in the form of a set of elements, divided into several hierarchical levels; V.M. Bondarenko [2] proposes, in particular, to consider 4 interrelated structural levels: material, section, structure (element), structural system of the building (structure);

10.8) consideration of the so-called characteristic volume, the properties of which determine the behavior of the entire structure or its individual element; this is a section of a rod of some length corresponding to the distance between cracks $l_{c r c}$ or conditionally assigned.

The cross section of the characteristic volume selected in this way is divided into elementary sections (areas), or section components, the deformation compatibility of which is established by the hypothesis of flat sections (possibly corrected taking warping into account [2]). With this approach, a change in bending moments along the length of the structure leads to a break in the rod adjacent members curvatures values.

This disadvantage is eliminated by using the finite element method on computer software, in which individual sections are not considered, and the entire structure is specified as a set of elements with certain properties. The hypothesis of flat sections is not used, and the compatibility of deformations is ensured by the neighboring elements' nodes displacements equality condition, due to which it is possible to obtain a continuous field of deformations along the length of the structure.

However, FEM has its disadvantages, described above, the totality of which outweighs the scale in favor of using a numerical diagrammatic method (based on a discrete deformation model), taking into account the principles 6.7 and 6.8 in nonlinear calculations. It should be noted that this method is a highly simplified analogue ofFEM, because the change in bending moments and longitudinal forces along the length of the element is neglected in it and the presence of transverse forces in most cases is not taken into account.

\section{Research results and their analysis}

The result of this research is a three-level computational model, hierarchically built according to the computational scheme discretization principle (according to the principle of Academician V.M. Bondarenko [2]):

Level 1 - "element" - a design model of the rod, built according to the well-known rules of structural mechanics (for example, according to the finite element method [13]); as a result of calculations at this level, forces are obtained in all sections of the rod;

Level 2 - "section" - improved nonlinear (discrete) deformation model (INDM), adopted according to BC 63.13330 with a number of the author's essential clarifications;

Level 3 - "material" - a design model of standard samples of concrete and reinforcement under the action of a central axial compressive or tensile static short-term load, or under the action of a static short-term load of pure shear; the behavior of the samples is described by the improved tension / compression diagrams of the professor N.I. Karpenko [14] or fundamentally new deformation diagrams for the corresponding loading, obtained by the author taking into account the accumulation of damage [15]. At the same time, the diagrams of concrete deformation during shear are built on the basis of theoretical results obtained by the author together with professor B.S. Sokolov [16], [17].

In the most general form, the interaction algorithm of these three different-level computational models is as follows: 
1. A geometric diagram of a rod element is built, constraints are applied, loads are applied, the necessary equations of mechanics are written down - a level 1 model is obtained "element".

2. At level 3 - "material" - are set by the properties of the materials used. Three diagrams are built for them: axial compression and tension, as well as shear.

3. At level 2 - "section" - are set by the initial characteristics of all cross-sections located along the length of the rod (geometry, reinforcement scheme, etc.).

4. Using the level 1 model, a static linear calculation of the rod is performed using one of the structural mechanics methods (for the purpose of automation, it is convenient to use FEM). Receive efforts in sections.

5. By efforts found using INDM the level 2 actual stiffness of all sections of the element can be calculated. In this case, nonlinearity is determined by the physical relationships of the level 3 model, as well as by the appearance and development of a crack in a given section.

6. For the statically indeterminate structures, taking into account the new stiffness, recalculate the forces in the sections at the 1 st level of modeling. The calculation in this case, as before in paragraph 3, is linear.

7. Again, as in Section 5, at the 2nd level, the stiffnesses of the sections are specified using a nonlinear calculation.

8. Items 6, 7 are repeated until the difference between the efforts (deformations) at the current and previous iterations is not less than $5 \%$.

9. After checks using the appropriate criteria for the onset of one or another limiting state, either the calculation is stopped, or, if necessary, is returned to the items 1 and 2 to change the initial properties of materials and the characteristics of the sections, respectively. And the algorithm will be repeated again.

In this case, the model of the 3rd level can be used independently when studying the properties of the material. Model of the 2 nd level - only in a compartment with the previous one - for calculating cross-sections. And the model of the 1st level includes 2 and 3 levels, it is used to calculate the structural element as a whole.

\subsection{Design model of the 1st level - "element"}

The design model of the 1st level rod element is built according to the general FEM rulesin the following sequence:

1. Let a rod of length be given $L$, under the action of an arbitrary system of forces (Fig. 1.1, a). The connections imposed at its ends can be any, while at one of the ends there may be no connections at all, then there will be a rigid termination at the second end. The section of the rod and the scheme of its reinforcement are also arbitrary.

2. We set the global coordinate system $O x y z$ starting at the left end of the rod: axis $z$ along the rod, axis $x$ and $y$ - are placed in the cross section (Fig. 1.1, b), so that the axes Oxyz will be the main center axes. The rod element is split into finite elements by nodes (FE): recommended at least $n=7$ nodes, including the extreme ones, so we get six FE. We number the nodes from left to right.

3. Load acting on a given FE, reduced to the nodal $R_{i}$ and $R_{j}$ (forces and moments), $i$, $j=1 \ldots n$ ( $i, j-$ the number of the first and second node, respectively). For this, the rod is considered to be elastic linearly deformable, which makes it possible to apply the principle of superposition (the principle of the forces action independence): the result of the forces system action is equal to the sum of the results obtained from the action of each force separately. 

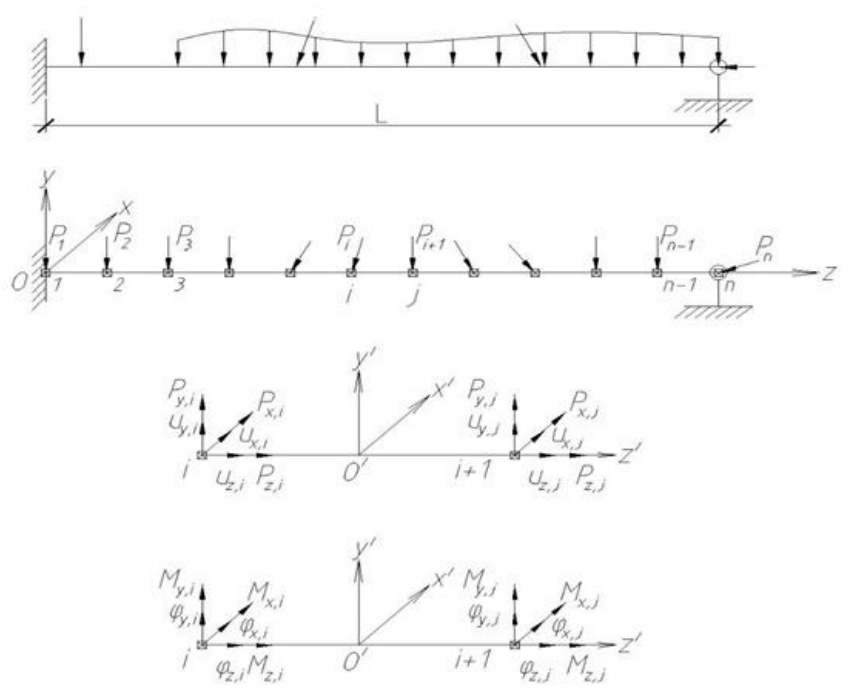

Fig. 1. Model of the 1st level - "element"

4. Moving everyone $i$-th not $u_{i}$ carried out in the direction of the force acting on it $R_{i}$, in magnitude, it depends on both $R_{i}$, and from the action of forces in neighboring nodes $P_{j}$. Moving $u_{i}$ is defined by the formula:

$$
u_{i}=\sum_{j=1}^{n-1} \delta_{i, j} R_{j}, i, j=1 \ldots n,
$$

where $\delta_{i j}$ is a moving $i$-th node from the action of a unit force $\bar{R}_{j}=1$.

In matrix form, the formula (1) is represented as $\{u\}=[\delta] \times\{R\}$. Formally, it is possible to write:

$$
\{R\}=[\delta]^{-1} \times\{u\},\{R\}=[K] \times\{u\},
$$

where $[K]=[\delta]^{-1}$ define the stiffness matrix which coefficients $k_{i, j}$ have the following physical meaning: it is power $P_{i}$, arising in $i$-th node under the action of force in $j$-th node causing a single movement in this node $\bar{\delta}_{j}=1, k_{i j}=\frac{R_{i}}{\bar{\delta}_{j}}$. At the same time, the very power in $j$-th node is equal to $k_{j, j}$.

Stiffness matrix $[K]$ is symmetrical. Mathematically this means $[K]=[K]^{T}$.

5. Let us select an arbitrary FE with node numbers $i$ (left) and $j$ (right). Let us introduce a local coordinate system for it $O^{\prime} x^{\prime} y^{\prime} z^{\prime}$ with the beginning in the rod middle (Fig. 1, c). We decompose the nodal forces and displacements into projections. We get the corresponding column vectors of the load and displacement:

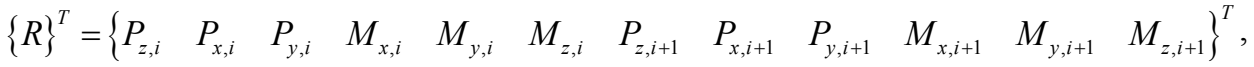

$$
\begin{aligned}
& \{u\}^{T}=\left\{\begin{array}{lllllllllllll}
u_{z, i} & u_{x, i} & u_{y, i} & \varphi_{x, i} & \varphi_{y, i} & \varphi_{z, i} & u_{z, i+1} & u_{x, i+1} & u_{y, i+1} & \varphi_{x, i+1} & \varphi_{y, i+1} & \varphi_{z, i+1}
\end{array}\right\}^{T} .
\end{aligned}
$$


FE has 12 degrees of freedom: three displacements $u_{x}, u_{y}, u_{z}$ and three turns $\varphi_{x}, \varphi_{y}$, $\varphi_{z}$ at each node. At the same time, the forces $P_{x}, P_{y}$ cause a cut, $P_{z}$ is the tension or compression, moments $M_{x}, M_{y}$ define bend, $M_{z}$ determines torsion.

Stiffness matrix of the element Fig. 1 has a dimension of $12 \times 12$ and includes the following quantities (the matrix itself is not given to reduce the volume of the article, for this see. [13]): $D_{z}$ is an axial stiffness of the element; $D_{x}$ is a bending stiffness about the axis $O x ; D_{y}$ is a bending stiffness about the axis $O y ; D_{k}$ is at orsional stiffness; $l$ is the finite element length.

6. We believe that along the length of the finite element its physical and mechanical properties do not change (with a reservation for transverse reinforcement, which is discussed below) and are determined by the properties of only one cross section. The formulas for determining the stiffness in the stiffness matrix in the most general case for reinforced concrete have the form:

$$
\begin{aligned}
& D_{z}=v_{b} E_{b} A_{b}+k_{b, a d} v_{b, a d} E_{b, a d} A_{b, a d}+k_{s} v_{s} E_{s} A_{s}+k_{s, a d} v_{s, a d} E_{s, a d} A_{s, a d}+ \\
& +k_{r 1} v_{s, r 1} E_{s, r 1} A_{s, r 1}+k_{r 2} v_{s, r 2} E_{s, r 2} A_{s, r 2} \\
& D_{x}=v_{b} E_{b} I_{b x}+k_{b, a d} v_{b, a d} E_{b, a d} I_{b x, a d}+k_{s} v_{s} E_{s} I_{s x}+k_{s, a d} v_{s, a d} E_{s, a d} I_{s x, a d}+ \\
& +k_{r 1} v_{s, r 1} E_{s, r 1} I_{s x, r 1}+k_{r 2} v_{s, r 2} E_{s, r 2} I_{s x, r 2} \\
& D_{y}=v_{b} E_{b} I_{b y}+k_{b, a d} v_{b, a d} E_{b, a d} I_{b y, a d}+k_{s} v_{s} E_{s} I_{s y}+k_{s, a d} v_{s, a d} E_{s, a d} I_{s y, a d}+ \\
& +k_{r 1} v_{s, r 1} E_{s, r 1} I_{s y, r 1}+k_{r 2} v_{s, r 2} E_{s, r 2} I_{s y, r 2} \\
& D_{k}=v_{b} G_{b} I_{b k}+k_{b, a d} v_{b, a d} G_{b, a d} I_{b k, a d}+k_{s} v_{s} G_{s} I_{s k}+k_{s, a d} v_{s, a d} G_{s, a d} I_{s k, a d}+ \\
& +k_{r 1} v_{s, r 1} G_{s, r 1} I_{s k, r 1}+k_{r 2} v_{s, r 2} G_{s, r 2} I_{s k, r 2} \\
& D_{Q}=v_{b} G_{b} A_{b}+k_{b, a d} v_{b, a d} G_{b, a d} A_{b, a d}+k_{s} v_{s} G_{s} A_{s}+k_{s, a d} v_{s, a d} G_{s, a d} A_{s, a d}+ \\
& +k_{r 1} v_{s, r 1} G_{s, r 1} A_{s, r 1}+k_{r 2} v_{s, r 2} G_{s, r 2} A_{s, r 2}
\end{aligned}
$$

where $E_{b}, E_{b, a d}, E_{s}, E_{s, a d}, E_{s, r 1}, E_{s, r 2}$ and $G_{b}, G_{b, a d}, G_{s}, G_{s, a d}, G_{s, r 1}, G_{s, r 2}$ are the initial deformation moduli and shear moduli, respectively, of the base concrete, added concrete reinforcement, main flexible rod reinforcement, added flexible rod reinforcement, rigid central reinforcement (steel core), rigid outer steel reinforcement (pipe shell); $A_{b}$, $A_{b, a d}, A_{s}, A_{s, a d}, A_{s, r 1}, A_{s, r 2}$ are the areas of the section corresponding components; $I_{b x}$ $, I_{b x, a d}, I_{s x}, I_{s x, a d}, I_{s x, r 1}, I_{s x, r 2}$ and $I_{b x}, I_{b x, a d}, I_{s y}, I_{s y, a d}, I_{s y, r 1}, I_{s y, r 2}$ are the moments of inertia of the corresponding components of the section about the axes $O x$ and $O y ; I_{b k}$, $I_{b k, a d}, I_{s k}, I_{s k, a d}, I_{s k, r 1}, I_{s k, r 2}$ are the torsional moments of inertia of the corresponding components of the section; $v_{b}, v_{b, a d}, v_{s}, v_{s, a d}, v_{s, r 1}, v_{s, r 2}$ arethe secant modulus 
coefficients (otherwise, the elastoplastic deformation coefficients) of the section corresponding components; $k_{b, a d}, k_{s} k_{s, a d} k_{r 1} k_{r 2}$ are the team work ratios.

The main determining parameter for the connection between the models of 1 and 2 levels is the section stiffness, therefore, in the author's publications, the patterns of its change, depending on all sorts of factors, are considered in detail. Wherein:

1 - all geometric characteristics of the section for the concrete part of the section are determined taking into account the development of cracks (i.e., minus the damaged part area);

2 - secant modulus coefficients $v$ are differentially determined for the compressed and stretched zone of the section, as well as for the case of shear according to the corresponding deformation diagrams of concrete and reinforcement (based on the model of the 3 rd level);

3 - the presence of transverse reinforcement is taken into account by averaging the concrete deformation modulus according to the formula:

$$
E_{b w}=E_{b}\left(1-\mu_{V w}\right)+E_{s} \mu_{V w},
$$

where $\mu_{V w}=\frac{n_{s w} \pi d_{s w}^{2} l_{s w}}{4 s_{w} A_{b 0}}$ is the volumetric transverse reinforcement ratio.

7. After the expressions for one finite element are built, they consider the rod element as a whole: form the global stiffness matrix $\left[K_{g}\right]$. The boundary conditions are set. The solution to the problem is to find the displacement vector $\{u\}$, which reduces to solving a system of linear algebraic equations by the matrix method:

$$
\{u\}=\left[K_{g}\right]^{-1}\{R\}
$$

$\left[K_{g}\right]^{-1}$ is amatrix inverse to $\left[K_{g}\right]$.

8. Based on the displacements found, all the components necessary for the SSS calculation are further determined: deformations, stresses and forces.

Once again, we note: the presented calculation apparatus of the 1 st level model is generally known, the scientific novelty in it is the connecting link between the models of the 1 st and 2 nd levels - the stiffness of the section: the formulas (3)-(7).

\subsection{Design model of the 2nd level - "section"}

The design model of the 2nd level - "section" - is an improved nonlinear deformation model of normal section BC 63.13330 with a number of refinements:

1 - all six components of external force factors acting on the element are considered (in BC 63.13330 there are only three of them: $\mathrm{N}_{\mathrm{z}}, \mathrm{M}_{\mathrm{x}}, \mathrm{M}_{\mathrm{y}}$ ): the main vector of forces $R$ definesthree $\left(N_{z}, Q_{x}, Q_{y}\right)$, at the main point $M$ definesthree $\left(M_{x}, M_{y}, M_{z}\right)$;

2 - the calculations take into account the work of the stretched zone of concrete above the crack;

3 - to describe the relationship between stresses and deformations of concrete, in addition to the diagrams of deformation for uniaxial tension / compression, shear diagrams are

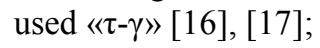

4 - when considering a steel-reinforced concrete element, the contact interaction between the steel of rigid reinforcement and concrete is taken into account (the degree of 
violation of contact interaction is still poorly understood, in a first approximation the issue is considered in the work [18]);

5 - in reinforcing case, a reinforced concrete element with a steel fiber concrete jacket, the contact interaction between the new concrete and the old one and the difference in their rheological properties are taken into account [19]-[21].

Thanks top. 3 it becomes possible to calculate the bending elements' sections in the joint action zone $M$ and $Q$ based on the diagrammatic method.

For transition to the section, a straight rod of arbitrary length 1 should be considered first. We focus on this special attention: NDM is not a sectional model, but a model of a rod of arbitrary length $\boldsymbol{l}$, it is just that the rod length is shortened during the conversion of the calculated values. Nevertheless, the name for the model of the 2 nd level was conventionally adopted as "section".

So, at the rod pole $O$, we put the right coordinate system $O x y z$ : axis $z$ - along the rod, axis $x$ - from drawing plane, axis $y$ - above. In this case, let the axes be the main central axes. At this pole, we also put the system of forces:

$$
\{P\}^{T}=\left\{\begin{array}{llllll}
N_{z} & Q_{y} & Q_{x} & M_{z} & M_{y} & M_{x}
\end{array}\right\}^{T} .
$$

These forces will cause the pole to move accordingly.:

$$
\{u\}^{T}=\left\{\begin{array}{llllll}
u_{z} & u_{y} & u_{x} & \varphi_{z} & \varphi_{y} & \varphi_{x}
\end{array}\right\}^{T}
$$

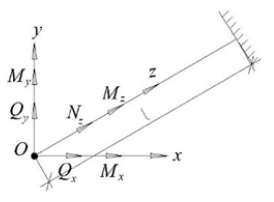

a)

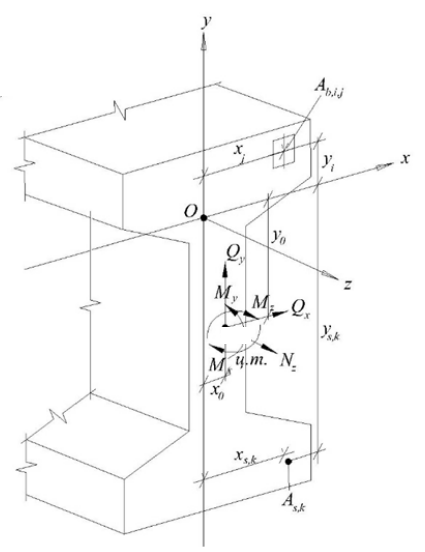

b)

Fig. 2. Model of the 2nd level - "section": $a$ - rod; $b$ - section

The efforts in formula (10) are taken based on the element calculation results at the previous modeling level.

Resolving the equations for the model of the 2 nd level in order to reduce the text, we write without output:

$$
\varepsilon_{z}=\frac{N_{z}}{D_{z}}, \theta_{z}=\frac{M_{z}}{D_{k}}, \chi_{x}=\frac{M_{x}+M_{y}}{D_{y}}, \chi_{y}=\frac{-M_{y}+M_{x}}{D_{x}},
$$


where $\varepsilon_{z}$ defines longitudinal relative deformations of the section at the level of the center of the selected axes; $\theta_{z}$ is are lative twist angle; $\chi_{x}, \chi_{y}$ define a curvature about the respective principal central axes.

A separate publication will be devoted to the output of these expressions.

\subsection{Design model of the 3rd level - "material"}

As a design model of the reinforcement, a rod sample was adopted according to GOST 12004, the behavior of which under load is described by a two-line Prandtl diagram according to BC 63.13330, which is not included here.

The design model of concrete in compression, tension and shear has its own distinctive features. In compression, the concrete model is a prism with dimensions of $150 \times 150 \times 600$ $\mathrm{mm}$, in tension - a prism with fillets, the prism section is $100 \times 100 \mathrm{~mm}$. The behavior of concrete under load is described by the improved diagrams of Academician N.I. Karpenko (they can be found in [14]) or by the author's curvilinear diagrams, taking into account the accumulation of damage [15]. In shear, the concrete model is a prism with the dimensions of $150 \times 150 \times 600 \mathrm{~mm}$, the behavior of which under load is described by a curvilinear diagram obtained by the author together with prof. B.S. Sokolov based on his theory [16], [17].

The formulas for describing the deformation diagrams of concrete in tension and compression have the form [15]:

$$
\sigma_{b t}=a_{t}\left(1-b_{t} D_{b t}\right)^{c_{t}} E_{b t} \varepsilon_{b t}, \sigma_{b}=a_{c}\left(1-b_{c} D_{b}\right)^{c_{c}} E_{b} \varepsilon_{b},
$$

where $\sigma_{b t}, \varepsilon_{b t}$ and $\sigma_{b}, \varepsilon_{b}$ are the current stresses and relative deformations in tension and compression; $a_{t}, b_{t}, c_{t}$ and $a_{c}, b_{c}, c_{c}$ are the design coefficients in tension and compression; $D_{b t}=\frac{\varepsilon_{b t}}{\varepsilon_{b t 2}}, \quad D_{b}=\frac{\varepsilon_{b}}{\varepsilon_{b 2}}$ are the corresponding deformation criteria of damageability; $\varepsilon_{b t 2}, \varepsilon_{b 2}$ are the ultimate deformations in tension and compression.

For the calculated coefficients, the following expressions are obtained analytically:

$$
\begin{aligned}
a_{t}=\frac{2,7 R_{b t}}{E_{b} \varepsilon_{b t 0}}, a_{c}=\frac{2,7 R_{b}}{E_{b} \varepsilon_{b 0}}, b_{t} & =\frac{1}{50 R_{b t}}, b_{c}=\frac{1}{50 R_{b}}, c_{t}=50 R_{b t} \frac{\varepsilon_{b t 2}}{\varepsilon_{b t 0}}-1, \\
c_{c} & =50 R_{b} \frac{\varepsilon_{b 2}}{\varepsilon_{b 0}}-1,
\end{aligned}
$$

where $\varepsilon_{b t 0}, \varepsilon_{b 0}$ are the relative deformations corresponding to vertex stresses; $R_{b t}, R_{b}$ define design resistance of concrete in tension and compression.

If for short-term loading we take the diagrams' parametric points according to $\mathrm{BC}$ 63.13330, namely: $\varepsilon_{b t 0}=0.0001, \varepsilon_{b t 2}=0.00015, \varepsilon_{b 0}=0.001, \varepsilon_{b 2}=0.0035$, then the following deformation diagrams curves of various classes concretes under uniaxial compression and tension can be obtained (ordinary concretes B7.5-B60 are shown, for high-strength concretes B65-B100 the graphs will be similar): 

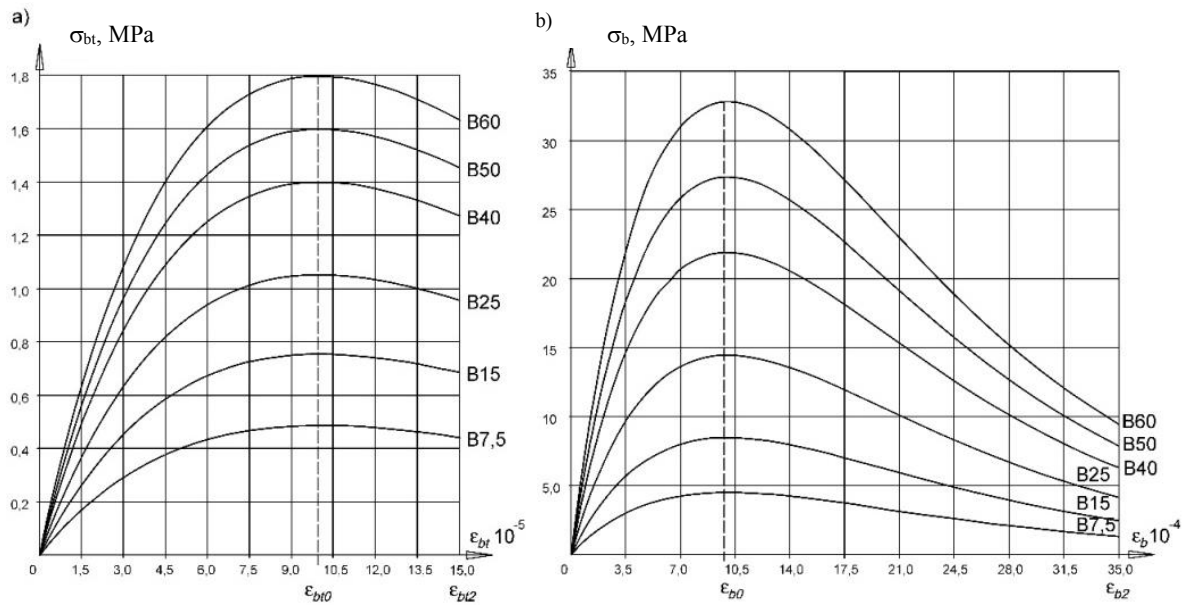

Fig. 3. Deformation diagrams of different strength classes concretes under uniaxial tension $(a)$ and compression (b), built by the formulas (13)

Fig. 3 shows the design diagrams that can be used in calculations for the $I$ group of limit states. Normative diagrams are constructed according to the same formulas (13), but with the replacement of the concrete calculated characteristics for the normative ones.

To describe the diagrams of concrete deformation in shear based on the theory of force resistance of anisotropic materials to compression according to professor B.S. Sokolov [16], [17], the expressions for the diagram parametric points are obtained $\tau_{b}-\gamma_{b}$ :

$$
\begin{gathered}
\gamma_{b 1}=\frac{\left(\varepsilon_{b 1}-0.5 k_{1} \varepsilon_{b t 2} \operatorname{ctg} \alpha\right) \sin \alpha}{0.4 k_{2}}, \tau_{b 1}=0.4 E_{b} \gamma_{b 1} \\
\gamma_{b 0}=\frac{\varepsilon_{b 0} \sin \alpha}{0.4 k_{2}}, \tau_{b 0}=R_{s h} ; \\
\gamma_{b 2} \approx \frac{\varepsilon_{b 2} \sin \alpha}{0.4 k_{2}}, \tau_{b 2}=\tau_{b 0}=R_{s h} .
\end{gathered}
$$

where $\varepsilon_{b 1}=\frac{0.6 R_{b}}{E_{b}}$ is the boundary of the compressed concrete work elastic stage.

To identify the relationship of the concrete deformation diagrams in compression, tension and shear with each other, we will superimpose all three of them on one coordinate plane (Fig. 4). To do this, for each diagram, we will take its own scale of the stress axis, and the tensile and shear deformations will be expressed in terms of compression deformations: $\varepsilon_{b t}=\mu \varepsilon_{b} \approx 0.2 \varepsilon_{b}, \gamma_{b}=\frac{\varepsilon_{b} \sin \alpha}{0.4 k_{2}}$. Then we get the graph in Fig. 4 .

In this figure, for diagrams 1 and 2 , the parametric points are indicated by the deformations, which are determined using the above-introduced dependencies: $\bar{\varepsilon}_{b t}=\frac{\varepsilon_{b t}}{0.2}$, $\bar{\gamma}_{b}=\frac{0.4 k_{2}}{\sin \alpha} \gamma_{b}$. Compression diagram 3 shows three characteristic areas:

I - area of joint resistance to compression of separation, shear and crushing zones;

II - area of exclusion of the separation zone from work and further joint resistance to compression of shear and crush zones; 
III - area of exclusion of the shear zone from work and further resistance to compression only of the crushing zone.

Fig. 4 also shows that the abscissas of most of the parametric points of the three deformation diagrams practically coincide (except $\bar{\gamma}_{b 0}$ ):

$-\bar{\varepsilon}_{b 11}=\bar{\gamma}_{b 1}$ is inelastic deformations onset in the separation zone;

- $\bar{\varepsilon}_{b t 0}=\varepsilon_{b 1}$ is transverse separation vertical cracks nucleation, their development, continuing up to deformations $\bar{\varepsilon}_{b t 2}$, after which the stretch zone is turned off from work (the boundary between regions I and II);

$-\bar{\gamma}_{b 2}=\varepsilon_{b 0}-$ after the shear zones are completely turned off, crushing of the concrete core begins (the border between regions II and III).

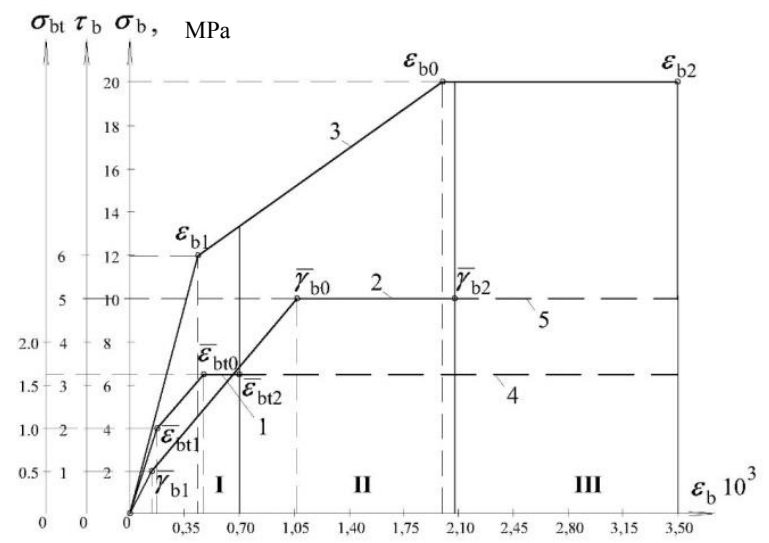

Fig. 4. Diagrams of series 5.1 cylindrical samples deformation [22] made of concrete of class B30 under tension - 1, shear - 2 and compression - 3; 4 - area of vertical cracks opening; 5 - resistance area due to frictional forces and engagement of concrete surfaces; I, II, III - areas of the element characteristic work at various loading stages

In this case, after reaching the value in the stretched deformation zone $\varepsilon_{b t}=\varepsilon_{b t 2}$ the tension diagram 1 continues in the form of a dotted section 4 , which characterizes the vertical cracks' opening. In addition, for shear diagram 2 after deformations $\gamma_{b}=\gamma_{b 2}$ dotted section 5 characterizes the work of friction forces and the engagement of concrete surfaces along inclined platforms.

In the end, we note that the models of all three levels are discrete, which is convenient for their automation and programming in computer software.

\section{Conclusion}

1. New concepts of theory, models and modeling, allowing a deeper understanding of the problem, have been introduced. The most general structure of the created theory of the diagrammatic method for calculating rod elements made of reinforced concrete has been developed.

2. A three-level design model has been proposed. It is hierarchically built according to the principle of discretization of the design scheme: level 1 - "element" - design model of the rod, built according to the well-known rules of structural mechanics; level 2 "section" - improved nonlinear (discrete) deformation model (INDM), adopted 
according to BC 63.13330 with a number of the author's essential clarifications; level 3 - "material" - a design model of concrete and reinforcement standard samples under the action of a central axial compressive or tensile static short-term load, or under the action of a static short-term load of pure shear. A detailed algorithm for the models' interconnection at all three levels is presented.

3. For the model of the 1 st level, the expressions for assessing the stiffness, which take into account the multicomponent cross-section, including the steel-fiber reinforced concrete cage, the steel core and the shell, as well as the conditions for the joint operation of the components are proposed.

4. In the design model of the 2 nd level - "section": 1 - all six components of external force factors acting on the element are considered (in BC 63.13330 there are only three of them); 2 - the calculations take into account the work of the concrete stretched zone over the crack; 3 - shear diagrams are used to describe the relationship between concrete stresses and deformations, in addition to uniaxial tension / compression deformation diagrams $\langle\tau-\gamma\rangle$ (Due to this, it becomes possible to calculate the cross-sections of bending elements in the zone of joint action of $\mathrm{M}$ and $\mathrm{Q}$ based on the diagrammatic method); 4 - when considering a steel-reinforced concrete element, the contact interaction between the steel of rigid reinforcement and concrete is taken into account; 5 - in the case of reinforcing a concrete element with a steel fiber concrete jacket, the contact interaction between the new concrete and the old one and the difference in their rheological properties are taken into account.

\section{References}

1. V.M. Bondarenko, V.S. Fedorov, ACADEMIA. ARCHITECTURE AND CONSTRUCTION 2, 103-105 (2013).

2. V.M. Bondarenko, Concrete and reinforced concrete 1, 24-27 (2002).

3. V.S. Fedorov, V.E. Levitsky, Prospects for the development of the construction complex, 2014.

4. A.B. Golyshev, V.Ya. Bachinsky, Concrete and reinforced concrete 6, 16-18 (1985).

5. B.S. Rastorguev, Concrete and reinforced concrete 3, 16-19 (1993).

6. A.V. Borovskikh, V.G. Nazarenko, Theory of the power resistance of compressed concrete structures (Information-publishing activities of RAACS, Moscow, 2000).

7. G.A. Geniev, V.I. Kolchunov, N.V. Klyueva, A.I. Nikulin, K.P. Pyatikrestovsky, Strength and deformability of reinforced concrete structures under beyond design impacts (Publishing house of the ASV, Moscow, 2004).

8. V.S. Fedorov, V.I. Kolchunov, A.A. Pokusaev, N.V. Naumov, Scientific journal of construction and architecture 4, 11-28 (2019).

9. V.N. Baykov, M.I. Dodonov, B.S. Rastorguev [et al.], Concrete and reinforced concrete 5, 16-18 (1987).

10. N.I. Karpenko, T.A. Mukhamediev, M.A. Sapozhnikov, Improvement of methods for calculating statically indeterminate reinforced concrete structures, 1987.

11. N.I. Karpenko, General models of reinforced concrete mechanics (Stroyizdat, Moscow, 1996).

12. A.I. Zvezdov, A.S. Zalesov, T.A. Mukhamediev, E.A. Chistyakov, Concrete and reinforced concrete 2, 21-25 (2002).

13. Yu.A. Okopny, V.P. Radin, V.P. Chirkov, Mechanics of materials and structures (Mashinostroenie, Moscow, 2002). 
14. N.I. Karpenko, O.V. Radaykin, Construction and reconstruction 3 (41), 10-17 (2012).

15. O.V.Radaykin, Bulletin of civil engineers, Saint Petersburg: Publishing house: Saint Petersburg state University of architecture and civil engineering 6, 71-78 (2017).

16. B.S. Sokolov, O.V. Radaykin, International journal for the calculation of civil and building structures 15 (3), 149-160 (2019).

17. N.I. Karpenko, B.S. Sokolov, O.V. Radaykin, Design of concrete, reinforced concrete, stone and reinforced stone elements and structures using diagram calculation methods: monograph (Publishing house of ASV, Moscow, 2019).

18. L.S. Sabitov, Yu.G. Konoplev, O.V. Radaykin, Bulletin of the Volgograd state University of architecture and civil engineering. Series: construction and architecture 1 (78), 345-355 (2020).

19. O.V. Radaikin, L.A. Sharafutdinov, Izvestiya KGASU 1 (39), 111-120 (2017).

20. O.V. Radaikin, L.A. Sharafutdinov, Izvestiya KGASU 1(47), 155-165 (2019).

21. O.V. Radaikin, L.A. Sharafutdinov, Bulletin of BSTU named after V. G. Shukhov 3, 34-45 (2020).

22. B.S. Sokolov, Theory of force resistance of anisotropic materials to compression and its practical application (Publishing house of the ASV, Moscow, 2011). 